\title{
Insulin induces oxidative stress in the vascular wall of patients with atherosclerosis independently of systemic insulin resistance: the regulatory role of DPP4 inhibition
}

Ioannis Akoumianakis ${ }^{1}$, Laura Herdman ${ }^{1}$, Marios Margaritis ${ }^{1}$, Rana Sayeed ${ }^{2}$, George Krasopoulos ${ }^{2}$, Mario Petrou ${ }^{2}$, Norbert Tennagels ${ }^{3}$, Paulus Wohlfart ${ }^{3}$, Keith M Channon ${ }^{1}$, Charalambos Antoniades ${ }^{1}$

\author{
${ }^{1}$ Division of Cardiovascular Medicine, Radcliffe Department of Medicine, University of Oxford, UK \\ ${ }^{2}$ Department of Cardiothoracic Surgery, Oxford University Hospitals NHS Trust, UK \\ ${ }^{3}$ Sanofi Aventis Deutschland GmbH, Diabetes Research, Insulin Biology, 65026 Frankfurt, Germany
}

Background: Insulin may have protective roles in vascular cells, but its vascular effects in patients with atherosclerosis are unknown. Dipeptidyl peptidase 4 (DPP4) inhibitors are a class of insulin-sensitising agents that may regulate vascular responses to insulin in humans. We attempted to determine the direct vascular effects of insulin in humans with atherosclerosis, and explore the effect of DPP4 inhibition on vascular insulin signalling.

Methods: The study included 613 patients undergoing coronary bypass surgery. Vascular segments (internal mammary arteries (IMA), saphenous veins (SV)) were collected and incubated with insulin glargine active metabolite M1 (insulin), insulin degludec (DEG) and human insulin (HI) (1-100 nM as stated), with or without pre-incubation with KR62436 (a DPP4 inhibitor) at $70 \mu \mathrm{M}$. Vascular superoxide (02.-) was quantified by lucigenin chemiluminescence, while nitric oxide bioavailability was evaluated by quantifying the vasorelaxations to acetylcholine. Circulating DPP4 activity was measured in fasting serum using commercially available kits. Downstream signalling was evaluated by Western blotting for protein phosphorylation.

Results: Insulin increased NADPH-oxidases-derived O2.- in vessels from patients with or without diabetes, an effect reversed in vascular segments from diabetic patients pre-treated with an oral DPP4 inhibitor (A). In contrast, insulin reduced O2.- in vessels of healthy mice used as controls (not shown). Circulating DPP4 activity was associated with increased vascular NADPH-oxidases activity in the study population (not shown), while ex vivo pre-incubation of human vessels with KR62436 (DPP4-i) reversed the effect of insulin on vascular O2.-, supressing NADPH-oxidases activity (B), improving eNOS coupling (C) and ameliorating endothelial dysfunction in human vessels (D). This was a class effect, replicated using DEG and HI (data not shown). DPP4 inhibition improved downstream insulin signalling by reducing insulin receptor substrate 1 (IRS1) phosphorylation at Ser307, a site linked to molecular insulin resistance (E). The vascular effects of DPP4 inhibition may be regulated via AMPK, since DPP4-i increased AMPK Thr172 phosphorylation while AMPK inhibition with compound C reversed the protective vascular effects of DPP4-I (F).

Conclusions: We demonstrate for the first time that insulin induces oxidative stress and endothelial dysfunction in vascular segments from patients with atherosclerosis, independently of systemic insulin resistance. This may partially explain the inability of insulin treatment to improve cardiovascular outcomes in patients with moderately elevated blood glucose. Pre-treatment with a DPP4 inhibitor restores local insulin sensitivity modulating the vascular responses to insulin. These findings suggest that vascular sensitisation may be crucial when treating of diabetic patients in secondary prevention. 


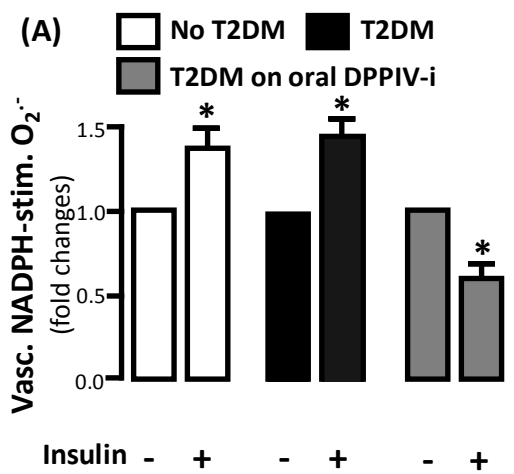

(D)

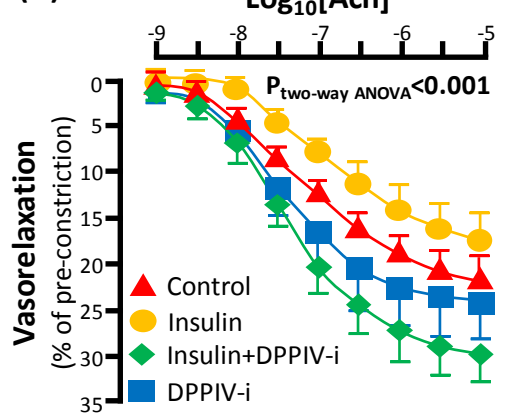

(B)

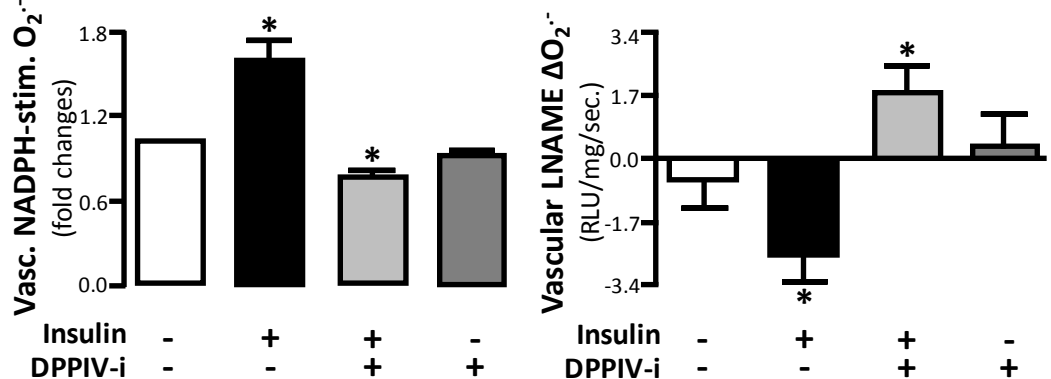

(E)

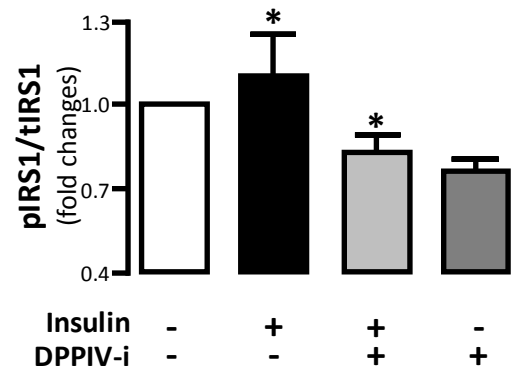

(C)

(F)

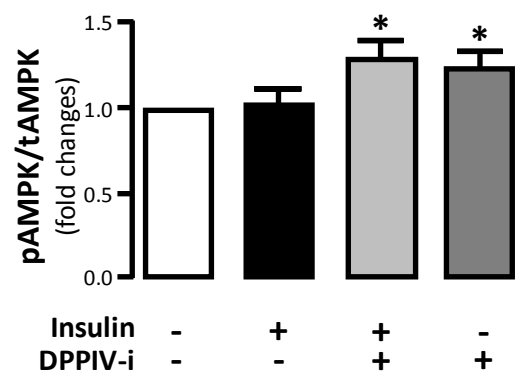

DPPIV-i = KR62436

Insulin=10nM

* $\mathrm{P}<0.05$ vs control 